\title{
Tangence
}

\section{Violette Leduc : écriture et sexualité}

\section{Catherine Viollet}

Numéro 47, mars 1995

Écritures au féminin : le genre marqué

URI : https://id.erudit.org/iderudit/025852ar

DOI : https://doi.org/10.7202/025852ar

Aller au sommaire du numéro

Éditeur(s)

Tangence

ISSN

0226-9554 (imprimé)

1710-0305 (numérique)

Découvrir la revue

Citer cet article

Viollet, C. (1995). Violette Leduc : écriture et sexualité. Tangence, (47), 69-83. https://doi.org/10.7202/025852ar d'utilisation que vous pouvez consulter en ligne.

https://apropos.erudit.org/fr/usagers/politique-dutilisation/ 


\title{
Violette Leduc : écriture et sexualité Catherine Viollet, CNRS, Paris
}

\author{
La littérature mène à l'amour, l'amour \\ mène à la littérature ${ }^{1}$. \\ J'ouvrirai le sexe d'Isabelle, \\ j'écrirai dedans avec mon encre bleue ${ }^{2}$.
}

L'érotisme et la sexualité (la sienne propre, mais également celle des autres) sont au cœur de l'œuvre de Violette Leduc. Une œuvre extrêmement riche, que l'on ne saurait certes réduire à ce seul thème et qui, bien qu'ayant déjà fait l'objet de nombreuses études $^{3}$, reste encore largement à découvrir. Cet aspect essentiel, novateur de son œuvre a valu à Leduc bien des déboires auprès des éditeurs et de certains critiques. Dans sa préface à $L a$ bâtarde, Simone de Beauvoir a su parfaitement définir les aspects contradictoires qui font précisément l'originalité de l'écriture de

1 Violette Leduc, La bâtarde, Paris, Gallimard, 1964, p. 399.

2 Id., La folie en tête, Paris, Gallimard, 1970, coll. • Folio •, p. 297.

3 Voir, par exemple, Mireille Brioude, La mise en scène du *je * dans l'cuure de $V$. Leduc, thèse nouveau doctorat, Université Paris VII, 1991; René de Ceccaty, La sentinelle du rêve, Michel de Maule, 1988; Ghyslaine CharlesMerrien, Violette Leduc ou le conps morcele, thèse nouveau doctorat, Université Rennes II, 1988, non publié; Isabelle de Courtivron, Violette Leduc, Boston, Twaine, 1988, et Androgyny, Misogyny and Madness. Three Essays on Women Literature, thèse Ph.D., Ann Arbor (Mich.), 1973; Alex Hugues, Violette Leduc. Motbers, Lovers and Language, W. S. Maney \& Son Ltd., 1994; Marie-Eेve Klein, Violette Leduc ou la revancbe du quotidien, thèse de $3^{\circ}$ cycle, Université Strasbourg II, 1983; Elaine Marks, - Lesbian Intertextuality •, George Strambolian et Elaine Marks (dir.), Homosexuality and French Literature: Cultural Contexts/Critical Texts, Ithaca (N. Y.)/Londres, Cornell University Press, 1979, p. 353-377; Masques, revue des homosexualités, $\mathrm{n}^{\circ}$ 11, 1981: -Violette Leduc*; Martha Noël Evans, "La mythologie de $l^{\prime}$ 'écriture dans La bâtarde de V. Leduc •, Littéralure, ${ }^{\circ}$ 46, mai 1982, p. 8292; Nord, $\mathrm{n}^{\circ}$ 23, juin 1994 : -Violette Leduc *; Adelaide Iula de Perilli, Contreesquisses: trois êtudes sur Violette Leduc, Rome, Bulzoni, 1991, et L'imaginaire baroque dans l'outure de Violette Leduc, thèse de doctorat, Paris, Sorbonne, 1993, 2 vol. Une biographie de Violette Leduc, par Carlo Jansiti, est actuellement en préparation, et devrait être publiée dans le courant de l'année 1995. 
Leduc en matière de sexualité et d'érotisme: à la fois "sincérité intrépide" et "brûlante intensité", "ingénuité morale" et "scrupuleuse honnêteté".

Dans sa manière d'aborder par l'écriture littéraire ce domaine essentiel de l'expérience humaine, cette "clé privilégiée du monde" "que constitue la sexualité, leduc me semble en effet occuper une place à part, une position pionnière, qui n'a sans doute pas encore été suffisamment mise en valeur. Les aspects et les expériences de la sexualité que son ouvre décrit, évoque ou met en scène sont multiples: une naissance illégitime et ses conséquences, des relations sexuelles tant avec des femmes qu'avec des hommes, un regard sur la prostitution, la découverte tardive du plaisir solitaire, l'avortement, des relations problématiques avec des homosexuels, l'inceste, et toujours l'observation aiguë d'autrui. Quel que soit l'aspect de ce vaste panorama qu'elle choisit d'explorer, avec une liberté de ton jusque-là inégalée, elle n'a de cesse de chercher le "mot justen, loin des approximations et des fausses pudeurs.

Mes recherches en critique génétique ${ }^{5}$ m'ont amenée à constater à quel point l'écriture sur la sexualité - en particulier lorsqu'il s'agit de ses formes les moins acceptées socialement - est soumise à tout un ensemble de pressions, de contrôles, de censure, auquel répondent certaines formes de transposition ou d'autocensure. Cette constatation, qui peut paraître a priori triviale, devient d'une complexité fascinante lorsqu'on a la chance d'avoir accès aux manuscrits, qui portent les traces concrètes du travail d'écriture, de production de l'œuvre, parfois de ses conditions mêmes de production. Précisons bien que ce n'est pas la sexualité en soi qui m'intéresse ici - à savoir l'expérience particulière qu'en a vécue tel ou tel écrivain (ceci est, tout au plus, l'affaire des biographes...) - mais la manière dont, en devenant objet du processus d'écriture, elle est représentée, transfigurée, mise en littérature. Car, écrit Leduc,

Le corps est secret... il prend, il ne vous rend rien. À toi de le rendre transparent. Peine perdue. Le corps ne se souvient pas. Ce sera du toc et de la fantaisie. [...] Pourquoi effaroucher?

4 Simone de Beauvoir, préface à La bâtarde, p. 16.

5 En particulier sur Marcel Proust, Ingeborg Bachmann, Francis Ponge, Thomas Mann. 
Pourquoi scandaliser? Présomptueuse, ouvre d'abord ton cahier. ${ }^{6}$

\section{Une initiation : L'asphyxie}

Dans le cas de Violette Leduc, le partage entre écriture et vécu n'est pas simple, du fait qu'il s'agit d'une œuvre largement autobiographique: son enfance, ses amours, ses rencontres, ses difficultés, sa vie au quotidien et son rapport au monde des objets, son activité d'écrivain en constituent explicitement la trame.

Venue à la littérature relativement tard (à trente-cinq ans, après avoir publié quelques textes journalistiques), elle lui consacrera désormais sa vie (le troisième volume de son autobiographie, La chasse à l'amour, ne paraîtra qu'après sa mort).

Son premier livre, L'asphyxie (1946), se présente sous forme de fragments transposés portant sur l'enfance et la prime adolescence (1907-1926), est plutôt centré sur les relations familiales mère et grand-mère. Il fourmille pourtant d'épisodes liés à la sexualité, où la narratrice n'est d'ailleurs pas toujours directement impliquée: la description de Fernand, le contrebandier, qui fascine la jeune narratrice, et avec qui elle dansera dans un estaminet; le comportement d'un homme qui s'intéresse à sa mère dans une fête foraine; Armandine, une camarade de classe, poursuivie par un exhibitionniste, qui attrappe "une fièvre de cheval à cause de ce membre inconnu" (p. 25); M. Pinteau, qui vit avec sa sœur ("On disait que le cher frère et la chère sœur...") et coince la petite fille sur ses genoux pour la déshabiller (p. 34). Vient ensuite le magistral avertissement de la mère à sa fille, à propos de sa naissance illégitime: "Écoute-moi... Essaie de comprendre. Si ça t'arrivait, une chose pareille, je te renierais. Moi, je ne savais rien. Le péché! C'est vague le péché! Si un homme t'appelait, n'y va pas. Suis ton chemin. Ne te retourne pas. Tu m'entends?" (p. 53). Quelques pages plus loin, la jeune adolescente assiste en voyeuse ("Dans le fournil où Mandine m'avait cachée, j'avais tout vu et tout entendu ", p. 71) à une scène d'approche sexuelle entre Mandine et un demi-vagabond, M. Dezaille. Puis à une conversation entre sa grand-mère et $M^{\text {me }}$ Barbaroux; cette dernière, obsé-

6 Violette Leduc, La folie en tête, op. cit., p. 297. 
dée par l'entretien de sa maison, évoque le comportement de son mari, les arguments qu'il utilise pour éviter les relations sexuelles ( .... Ce n'est pas raisonnable... On sera fatigué demain... I...] On attrapera un rhume... Comme tu es exigeante... N'y pense pas...", p. 95). Vient ensuite l'épisode de Mme Panier, dont le mari a en permanence les mains gantées de cuir noir, et caresse le mur autour du lit conjugal parce que, dit-elle, "il abusait, et que ces mains "nous auraient fait perdre la raison à tous les deux * (p. 107). La grand-mère raconte à son tour à sa petite-fille comment son mari l'a trompée sous ses propres yeux: "Je l'avais emmené chez mes amies, des jeunes filles. [...] Dans leur belle pièce, j'ai vu qu'il embrassait la plus gracieuse. Je le voyais dans la glace... [...] L'amour, ce n'est pas notre destin." (p. 117). Curieuse, l'adolescente a déjà un regard critique, non dépourvu d'humour: "Je tombai aussi sur le commis épicier. Il était étendu sur une demoiselle. Ils avaient chacun une pâquerette à la bouche. Chacun mâchonnait sa tige. Ils semblaient mécontents, mal à l'aise, irrésolus, poussifs. Ils soufflaient fort et les fleurs avançaient, reculaient... L'ensemble manquait de grâce." (p. 123). Et enfin la narratrice est fascinée par cette scène hallucinante un homme dans le jardin public, amoureux de la statue qui représente sa sœur ("Quand il s'agit d'elle, j'ai tous les droits...", p. 157 - , qui culmine en une crise d'épilepsie qu'elle compare à l'extase amoureuse: "Son visage fut éclairé de l'intérieur. Il reflétait une émotion somptueuse" (p. 161).

Ainsi, dès cette première ouvre sur l'enfance, le parcours initiatique en matière de sexualité - plus exactement, si l'on y regarde de près, d'hétérosexualité sous ses divers aspects découvre un panorama passablement "asphyxiant". Pour ce qui est du domaine affectif, l'absence du père qui refuse son existence, la relation conflictuelle et tourmentée entre la mère "irréprochable" et sa fille - que résume parfaitement le lapidaire incipit "Ma mère ne m'a jamais donné la main..." - ont pour contrepoint l'amour chaleureux et tendre qu'offre à l'enfant sa grand-mère, "l'ange Fidéline", et l'adoration réciproque qui les unit. Ce qui frappe dans L'aspbyxie, ce n'est pas seulement la multitude de situations évoquées, mais aussi le fait que l'enfant est constamment confrontée à un discours, parfois à mots cou- 
verts mais le plus souvent explicite, sur la sexualité — un sujet de conversation parmi d'autres, pas plus tabou qu'un autre.

\section{Une sexualité omniprésente}

Omniprésents, l'amour, le désir et la sexualité vont désormais habiter l'ensemble de l'œuvre. L'affamée (1948) et Trésors à prendre (1960), le journal d'un voyage dans le Sud de la France, sont hantés par - et consacrés à — la passion, dont l'érotisme est. sublimé à travers l'écriture, de Violette Leduc pour Simone de Beauvoir. Entre les deux, elle écrit ce qui deviendra plus tard Thérèse et Isabelle d'une part, et Ravages d'autre part, alors conçu comme un seul livre relatant l'itinéraire amoureux de la narratrice, Thérèse ${ }^{7}$. D'abord la rencontre avec Isabelle au collège, la découverte et le partage de l'univers érotique féminin; puis la période de vie commune avec Hermine, où s'inscrit la rencontre avec Marc, qui deviendra le mari de Thérèse. Ravages est publié en 1955, après que Gallimard en ait expurgé la première partie, qui paraîtra sous le titre de Thérèse et Isabelle seulement onze ans plus tard, en 1966. Avec la publication de La batarde (en 1964) commence la trilogie autobiographique, mais, surtout, Leduc obtient enfin auprès du grand public le succès qui s'était jusquelà fait attendre, et dont l'absence la désespérait ${ }^{8}$. C'est d'ailleurs à la fin de La bâtarde qu'elle raconte sa venue à l'écriture, comme à contre-cœur, sur les injonctions de Maurice Sachs ${ }^{9}$. Les deux autres volumes de l'autobiographie - La folie en tête (publié en 1970, et couvrant la période 1944-1954) et La chasse à l'amour (publié en 1973, un an après sa mort, et couvrant la période de 1954 à 1964) - relatent, parallèlement aux événements vécus, au récit des rencontres, des relations amoureuses et de la solitude, le travail d'écriture tel qu'il s'inscrit au fil du quotidien. Dans les nouvelles également, la sexualité tient une place de choix, qu'il

7 Thérèse est le premier prénom d'état civil de Violette Leduc.

$8 \quad \ldots$ bien qu'elle ait bénéficié jusqu'alors de l'estime de certains écrivains, et non des moindres - Genet, Cocteau, Jouhandeau, Sarraute - en plus du soutieri permanent et actif de Simone de Beauvoir.

9 - Maurice m'a dit le lendemain:

- Vos malheurs d'enfance commencent de m'emmerder. Cet après-midi vous prendrez votre cabas, un porte-plume, un cahier, vous vous asseoirez sous un pommier, vous écrirez ce que vous me racontez.

- Oui Maurice, dis-je, vexée.. (La bâtarde, p. 399) 
s'agisse de La vieille fille et le mort (1958), où l'écriture est la transfiguration d'un amour impossible ${ }^{10}$, des Boutons dorés, de La femme au petit renard ou encore du $i$, dialogue érotique au parfum d'inceste adelphique.

Omniprésente, la sexualité ne l'est pas seulement dans le monde des humains. Elle l'est aussi dans l'univers des objets, des plus quotidiens et des plus infimes (savon, peigne en écaille, serpillère ou dentelle de la chemise de nuit), comme elle habite les paysages et les monuments. À travers la relation que le corps établit avec lui, le monde se pansexualise. Ainsi l'approche de la cathédrale d'Albi, dont Simone de Beauvoir avait recommandé la visite, présente certaines caractéristiques d'une rencontre amoureuse:

Je tourne en rond sur la place, je tremble parce que moi touriste, je vais me fiancer à un monument et que je ne me décide pas et qu'il est trop tard pour reculer. Je la fuis. C'est si vite défloré avec l'œil la construction. ${ }^{11}$

En projetant sur les paysages qu'elle traverse son amour pour Simone de Beauvoir, Leduc les recrée à neuf, les métamorphose. Lors de la visite de l'église de Cordes, la contemplation esthétique du vitrail se transforme en un long moment d'effusion érotique, à travers les métaphores de la jouissance charnelle:

Long rendez-vous avec la rosace de l'église de Cordes [...]. Le rouge, le violet, le cyclamen se dilatent pour que mes yeux les prennent. [...] Le sexe de mes yeux n'est pas blessé à la surface du vitrail quand je les pénètre, chair fondante, chair profonde, matrice du secret, tombeau affolant de la surface peinte pour les deux sexes de mes deux yeux. [...] Concentrés énigmatiques, couleurs du vitrail, soleils du velours, qui-vive de mes orgasmes. [...] Quand je vous pénètre, calice répandu sur la vitre, je me creuse dans mes yeux. ${ }^{12}$

Dans les deux cas, c'est le regard qui opère la transmutation de la matière en jouissance pure: qui déflore, prend, pénètre et,

10 - Lecteur [...] tu pourrais te dire: Maurice Sachs, c'est donc le mort? Tu te tromperais. Le mort est un autre homosexuel que j'ai aimé, le mort est un homme riche et en bonne santé que j'ai changé en vagabond parce que mes doigts tenant le porte-plume pouvaient fermer les paupières d'un vagabond. (La bâtarde, p. 371). D'autres ont également vu dans ce récit une manière de sublimer l'absence du père.

11 Violette Leduc, Trésors à prendre, Paris, Gallimard, coll. • Folio •, 1960, p. 151.

12 Ibid., p. 200. 
"se creusant", mène à l'orgasme ${ }^{13}$. Contempler un monument ou décrire les objets les plus quotidiens à travers le prisme d'émotions érotiques, c'est faire de ces objets des acteurs à part entière dans la vie de la narratrice, des complices de ses affects, liés au corps par toutes les fibres de la sensualité ${ }^{14}$.

Complices d'une sexualité quasi universelle, les objets, à commencer par les plus humbles, se font également complices de l'écriture: En est témoin cet extraordinaire dialogue avec les paillettes de mica incrustées dans le sol du métro, à la fois "diamants " et "ciel étoilé":

Toi écrire oh! la la parions-en chuchotèrent dans mes yeux les paillettes d'un escalier du métro. Tu passais, nous existions. C'était cela te mettre à écrire avec tes petits yeux. Maintenant que tu nous vois, tu te prends au sérieux. Je vous décrirai. Tu n'en seras pas capable. C'est vrai, tu ne nous voyais pas. Tu commences à nous exploiter. [...]. ${ }^{15}$

\section{Une écriture sexualisée}

Pour Violette Leduc, l'acte d'écrire et l'acte d'aimer se confondent souvent, s'entremêlent de diverses façons:

Écrire. L'amour après l'amour. Je me courbais, je me redresse: mon désir de mieux écrire. ${ }^{16}$

Aimer, c'est écrire : les cahiers qu'elle montre régulièrement à Simone de Beauvoir sont non seulement le prétexte de rendezvous et de rencontres renouvelées, mais aussi et surtout l'occasion pour Violette Leduc d'exprimer, à travers les textes qui couvrent ces cahiers qu'elle soumet à la lecture de Beauvoir, cette passion dont ils sont, pour cette dernière, la seule forme acceptable. Ce seront L'affamée, Trésors à prendre et, dans une moindre mesure, La folie en tête.

13 On notera que Violette Leduc mêle et confond des points de vue traditionnellement définis comme féminins ou masculins, donc dissociés: la touriste se fiance à un monument qu'elle risque de déflorer... • Quand je vous pénètre, je me creuse- associe en une métaphore androgyne deux gestes, deux mouvements censés être exclusifs l'un de l'autre.

14 Voir l'aricle de Ghyslaine Charles-Merrien, - Le rôle des objets dans l'univers de Violette Leduc •, Nord, $\mathrm{n}^{\circ} 23$, juin 1994.

15 Violette Leduc, La bâtarde, op. cit., P.311

16 Violette Leduc, La folie en tête, op. cit., p. 117. 
76

Si aimer veut dire écrire, l'inverse aussi sera vrai: écrire, c'est manifester, mettre en jeu une relation amoureuse, érotique vis-àvis de la langue et - à travers elle - des objets et des êtres. Ainsi les sensations éprouvées au moment de l'écriture s'inscrivent-elles tout naturellement dans le registre de la sexualité :

Parfois je rêve à des aphrodisiaques que ma plume absorberait, les mots seraient amoureux de moi. J'aime d'amour les adjectifs, il ne faut pas en abuser, je passe outre. Qualifier, c'est prendre dans ses bras un absent. [...] Je me donne aux adjectifs, je meurs de plaisir pour eux. [...] Ouvrir une agence matrimoniale. Je marierais du matin au soir les adjectifs avec les sons, les couleurs et les parfums. ${ }^{17}$

Relation d'amour réciproque entre l'écrivain et la langue, dont Leduc ne cesse de souligner l'aspect charnel, ces "caresses", ces "étreintes", ces "corps à corps de la plume avec le papier ", de l'écrivain avec les mots; tout comme elle ne cesse de souligner la matérialité du processus d'écriture - une sorte d'artisanat auquel elle se sent désespérément vouée, et qu'elle compare fréquemment aux travaux du ménage:

- Ménagère pendant que j'écris. Écrivain pendant que je lave le parquet. ${ }^{18}$

C'est encore aux métaphores de la sexualité — vénale cette fois - que Leduc, non sans humour, a recours pour souligner les ambivalences de l'écriture, et exprimer sa lucidité face à la tentation du compromis pour plaire au lecteur, à l'éditeur :

Écrire, c'est se prostituer. C'est aguicher, c'est se vendre. [...] Chaque mot est une passe. Adjectif, tu viens? Dis, tu viens, chéri? Je te ferai des choses, adjectif, tu monteras au ciel. Combien? Le prix du livre à paraître. ${ }^{19}$

Dans sa mise en scène d'un dialogue entre écriture et sexualité, Violette Leduc innove. De la manière la plus directe, la plus évidente, la plus concrète qui soit, avec une liberté de ton jusque-là inconnue chez les écrivains de son sexe. Par exemple, lors d'un épisode où, précisément, elle cherche à capter sous sa

17 Ibid., p. 72-73. Déroutante Violette Leduc! Contrairement à ce que l'on pourrait croire, elle n'abuse effectivement guère des adjectifs, et vérification faite ils restent remarquablement rares dans ses textes; la vigueur du rythme y est beaucoup plus souvent liée aux verbes.

18 Violette Leduc, La chasse à l'amour, Paris, Gallimard, Gallimard, 1973, p. 296.

19 Violette Leduc, La folie en tête, op. cit., p. 412. 
plume la passion ô combien charnelle qui unit Thérèse et Isabelle, ces deux activités se confondent:

J'écrivais. J'écrivais sous leur dictée. J'écrivais d'une main et, de l'autre... je m'aimais pour les aimer, pour les retrouver, pour les traduire, pour ne pas les trahir. ${ }^{20}$

\section{Thérèse et Isabelle: questions de genèse}

Parmi les divers aspects de la sexualité que Leduc aborde dans son œuvre, il en est un qui se heurtera plus que tout autre à la censure de l'éditeur (Gallimard), et dont la publication restera bloquée pendant onze ans: il s'agit de la rencontre amoureuse et sexuelle de deux collégiennes, dont le récit sera finalement publié sous le titre de Thérèse et Isabelle. Dans la conception de l'auteur, cet épisode constituait la première partie du volume intitulé Ravages, qui retrace l'itinéraire amoureux de Thérèse ${ }^{21}$. À cette initiation passionnée succède une relation avec une autre femme (Cécile dans Ravages, Hermine dans La bâtarde), puis avec un homme (Marc dans Ravages, Gabriel dans La bâtarde), qui s'achève sur le récit de l'avortement de Thérèse.

C'est en 1948 que Violette Leduc entreprend la rédaction de Ravages. L'épisode "Thérèse et Isabelle semble être achevé dès 1951, tandis que la rédaction du volume se poursuit jusqu'en 1954. Beauvoir apporte alors à Queneau, chez Gallimard, l'ensemble du roman, mais la réaction de l'éditeur se fait attendre. Queneau n'aime pas la première partie, il pense (rapporte Simone de Beauvoir à Violette Leduc) qu'elle *alourdit inutilement l'ensemble du roman", et "rend le livre impossible à publier ouvertement"22. Les sous-entendus de l'expression *impossible à publier ouvertement" seront précisés dans une lettre ultérieure de Beauvoir qui, poursuivant les tentatives de négociations avec Gallimard, a présenté le volume à un second lecteur, J. Lemarchand. Ce dernier intervient

20 Ibid., p. 457.

21 L'épisode de la rencontre amoureuse entre Thérèse et Isabelle sera repris, avec des variantes, dans La bâtarde, et en constitue le troisième chapitre (p.76-109).

22 Lettre inédite de Simone de Beauvoir à Violette Leduc, non datée $15 \mathrm{mai}$ 1954, coll. C. Dehous], citée par Carlo Jansiti dans - Ils ont refusé le début de Ravages. C'est un assassinat., extrait d'une biographie en cours, Nord, $\mathrm{n}^{\circ} 23$, juin 1994, P. 82. 
de manière encore plus radicale, et "suggère une publication à part, sous le manteau" de la première partie, qu'il aime beaucoup $^{23}$. En fait, il juge le livre "d'une obscénité énorme et précise - et qui attirerait les foudres de la justice ${ }^{24}$. Pour ce qui est de "l'histoire des collégiennes", il demande finalement à l'auteur d'en "supprimer l'érotisme", tout en "gardant l'affectivité "25. Ce qui revient à un massacre pur et simple, et on comprend que Violette Leduc soit sortie "brisée" de l'entretien, et en soit littéralement tombée malade. Si l'ensemble de Ravages choque ces premiers lecteurs (mâles), ce sont les scènes d'érotisme entre les deux adolescentes qui font l'objet de la censure la plus sévère ${ }^{26}$. Certes, ce n'est pas la première fois qu'un tel sujet déclenche les foudres des censeurs ${ }^{27}$. Et pourtant, en 1951 est sorti le film de Jacqueline Audry Olivia, qui met en images le roman du même nom ${ }^{28}$. Si le cadre (un collège) et le sujet (l'attirance de la jeune élève Olivia pour son professeur $\mathrm{M}^{\text {lle }}$ Julie) sont tout à fait proches de ceux de Thérèse et Isabelle, la manière d'en traiter est beaucoup plus allusive, et se limite strictement au plan des sentiments. Il en est de même pour une autre œuvre célèbre qui traite du même sujet et s'achève de manière tragique, Jeunes filles en uniforme de Christa Winsloe (1930), dont Léontine Sagan réalisa la première version filmée. Il est fort probable que Violette Leduc a eu connaissance de ces deux cuvres; elle a lu l'œuvre de Colette — on pense bien sûr aux Claudine -, qu'elle trouve "timorée", trop "timide du point de vue érotique", ainsi que Poussière de Rosamond Lehmann, dont elle parle, en revanche, en des termes plutôt élogieux:

23 Lettre inédite non datée de Simone de Beauvoir à Violette Leduc 11 mai 1954, coll. C. Dehous], citée par Carlo Jansiti, ibid., p. 83.

24 Archives Gallimard, cité par René de Ceccaty dans Violette Leduc. Eloge de la Bâtarde, Paris, Stock, 1994, p. 17.

25 Lettre inédite de Leduc à Beauvoir, 16 mai 1954, citée par Carlo Jansiti, ibid., p. 83.

26 Mais pas exclusivement : certaines phrases décrivant le sexe masculin sont remplacées par des points de suspension, et il est derriandé que l'épisode de l'avortement soit remanié.

27 Voir par exemple l'ouvrage d'Yvan Leclerc, Crimes écrits. La littérature en procès au $19^{\circ}$ siècle, Paris, Plon, 1991, et le procès - pour obscénité - de Radclyffe Hall (1928), dont Le puits de solitude fut publié, par Gallimard d'ailleurs, en 1946. Carlo Jansiti rappelle également que le roman de Patricia Highsmith The Price of Salt, publié en 1952 aux Etats-Unis sous le pseudonyme de Claire Morgan, fut refusé en France pour des raisons analogues; il n'y sera publié que 33 ans plus tard, sous le titre Carol, Paris, Stock, 1985.

28 Olivia par Olivia, Londres, The Hogarth Press, 1949; trad. fr. Stock, 1951. 
Le soir j'ai été plus modeste grâce aux bonnes feuilles du premier roman de Rosamond Lehmann. Deux adolescentes s'aimaient, une femme osait l'écrire. Un doux soleil ruisselait en mélancolie, un personnage musclait l'histoire : Jennifer. Aimezvous Jennifer? [...] Nous parlions d'elle dans les couloirs, dans les bureaux $[\ldots]{ }^{29}$

Ce qui différencie Thérèse et Isabelle de la plupart des œuvres écrites jusque-là sur l'homosexualité féminine par des écrivains de sexe féminin ${ }^{30}$, c'est un érotisme à la fois poétique et réaliste, à la fois lyrique et précis. La nouveauté absolue du ton, sa beauté tiennent probablement à cette association des extrêmes. L'amour réciproque, la jubilation partagée que décrit Thérèse et Isabelle s'oppose également à la plupart des œuvres portant sur ce sujet parce qu'elle ne s'achève pas de manière tragique: la séparation des jeunes amantes est le fait du hasard ${ }^{31}$ :

- Nous ne nous quitterons pas?

- Nous ne nous quitterons pas.

Le mois suivant, ma mère me reprit. Je ne revis jamais Isabelle. ${ }^{32}$

Mais ce qui frappe avant tout, c'est l'évidence, la fougue passionnée, l'innocence qui se dégage de ce récit et que ne vient troubler ni contrarier le moindre sentiment de culpabilité, ce qui en exclut toute résonance obscène.

[...] le début de Ravages n'est pas sale. Il est vrai. Il salira celui qui veut être sali. C'est de l'amour, ce sont des découvertes. Thérèse et Isabelle sont toutes neuves. Elles s'aiment dans un collège pendant trois jours et trois nuits. Elles ne voient pas le mal. La censure le verrait-elle où il n'est pas? Thérèse et Isabelle sont trop authentiques pour être vicieuses. Il n'y a pas de vices. ${ }^{33}$

La censure qu'infligent à Violette Leduc les éditions Gallimard (mais aussi d'autres éditeurs à qui le volume avait été proposé)

29 Violette Leduc, La bâtarde, op. cit., p. 159.

30 Après la publication de Thérèse et Isabelle (1966), l'écriture de la sexualité entre femmes se fait plus audacieuse, ose en dire davantage. ainsi que l'avait souhaité Violette Leduc: que l'on pense notamment aux Guérillères (1969), et plus tard au Conps lesbien (1973) de Monique Wittig.

31 Alors qu'en fait Violette Leduc et sa compagne furent expulsées du collège après avoir été dénoncées (voir La bâtarde, p. 116-118).

32 Ibid., p. 120.

33 Violette Leduc, La chasse à l'amour, op. cit., p. 21. 
80

en refusant de publier la partie "Thérèse et Isabelle "de Ravages $^{34}$ prend les proportions d'une véritable "catastrophen, qui menace gravement l'équilibre et la santé de l'écrivain en provoquant une dépression profonde. Cet "assassinat", ainsi qu'elle le désigne, atteint le coeur même de son activité d'écrivain:

Mon encre: du plasma; ma plume: un cordon ombilical. Mon texte dactylographié : un nouveau-né. La censure a tout zigouillé. ${ }^{35}$

Ravages - qui, dans la construction finale imposée par l'éditeur, commence avec la rencontre entre Thérèse et Marc, se poursuit par la description de la relation entre Thérèse et Cécile, puis du trio Thérèse-Cécile-Marc, et s'achève sur le récit de l'avortement de Thérèse - sera en fin de compte une œuvre mutilée, irrémédiablement amputée par rapport à la conception initiale qui retrace l'ensemble d'un itinéraire, avec ses différents moments:

Ma construction était solide. Ma construction s'écroule. La censure a fait tomber ma maison du bout du doigt. 36

C'est en vain que Simone de Beauvoir essaiera de défendre le récit dans son intégrité, et Violette Leduc exprime longuement sa révolte, son désarroi et son incompréhension vis-à-vis de la censure opérée par les éditions Gallimiard, alors qu'elle dit s'être efforcée avant tout de rendre "l'exactitude des sensations *

Pourquoi ne s'explique-t-on pas avec la censure? Pourquoi ne puis-je rencontrer les éditeurs? Je plaiderais la cause de mes deux illuminées. [...] Je la convaincrai. Ce ne sont pas de vilaines gamines. Ce sont mes hérö̈nes. Mes cent cinquante premières pages ne sont pas malpropres. Elles ne peuvent pas l'être. C'est ce qui a été. [...] J'ai savonné, lessivé, rincé à grande eau mes adjectifs et mes comparaisons. Mon labeur, vieille bornée, a la fraîcheur de la pâte dentifrice. ${ }^{37}$

34 Pour les circonstances précises de la publication, voir l'article de Carlo Jansiti (1994). En 1955, Jacques Guérin, mécène et ami de Violette Leduc, finance une édition privée ( 28 exemplaires) de Thérèse et Isabelle. Ainsi qu'elle le raconte dans La chasse à l'amour, Violette Leduc fabriquera sur la suggestion de Jacques Guérin, pour cette édition de luxe, un - faux-vrai * manuscrit établi à partir de la dactylographie de Ravages. Le texte publié par Gallimard en 1966 sous le titre Thérèse et Isabelle différe et de la version de Ravages et du manuscrit édité par Guérin.

35 Violette Leduc, La chasse à l'amour, op. cit., p. 20-21.

36 Ibid., p. 21.

37 Ibid., p. 22. 


\section{Vers une étude des manuscrits de Ravages}

Dans de telles circonstances, on comprend l'intérêt que pourront présenter pour la recherche les manuscrits, les avant-textes de l'œuvre de Violette Leduc ${ }^{38}$. Le dossier qui concerne Ravages comporte onze cahiers d'écolier, numérotés par Leduc. À ces cahiers manuscrits s'ajoute une centaine de feuillets, dactylographiés ou manuscrits, dans des chemises, non classés, utilisés le plus souvent recto verso et numérotés sans ordre apparent. Il existe en outre une dactylographie partielle (en feuillets, effectuée par la dactylographe de Simone de Beauvoir), d'autres feuillets détachés d'un cahier vers 1961, une version dactylographiée définitive, des petits carnets portant des notes de relecture (dont beaucoup de pages ont été arrachées), et enfin des épreuves offertes à Jacques Guérin, datées, portant des corrections allographes.

Les cahiers d'écolier présentent une version beaucoup plus longue que celle du texte imprimé, avec des différences importantes, en particulier en ce qui concerne certaines scènes érotiques; la version imprimée a été, sans aucun doute, édulcorée.

À cette description sommaire des matériaux génétiques, il faut ajouter que les cahiers manuscrits de Ravages sont beaucoup plus épais qu'à l'origine, car l'écrivain a superposé, en les collant soigneusement, au recto comme au verso, plusieurs pages les unes sur les autres - pages arrachées à d'autres cahiers identiques. Ainsi le nombre de pages d'origine se trouve-t-il finalement multiplié par trois ou quatre, transformant chaque cahier en une sorte de "millefeuille". Étudier de manière systématique les différentes couches d'écriture nécessitera donc de décoller au moins partiellement ces pages, opération rendue extrêmement délicate par la fragilité du papier ${ }^{39}$. Ces cahiers portent de nombreuses corrections - variantes d'écriture ou de lecture - et très fréquemment des mentions du type "à revoir", "à refaire" (au

38 Carlo Jansiti m'a autorisée à consulter ces dossiers manuscrits et m'a permis de bénéficier de ses nombreux conseils. Qu'il en soit ici chaleureusement remercié.

39 D'après quelques sondages que j'ai effectués en décollant plusieurs épaisseurs de papier superposées, il semble que les feuillets collés les uns sur les autres correspondent grosso modo au même passage du texte - donc à différentes campagnes d'écriture, effectuées sur d'autres cahiers et rapportées sur le spremier - cahier. 
82

crayon), de larges biffures, ainsi que des corrections de la main de Simone de Beauvoir.

Ils comportent de nombreux passages inédits, et en particulier un épisode qui semble jouer un rôle-clef dans l'itinéraire amoureux de Thérèse (et correspond sans doute à une expérience partagée par bien des adolescentes): son premier geste érotique. La lenteur, les tâtonnements et la difficulté du processus de remémoration ne font que souligner son importance:

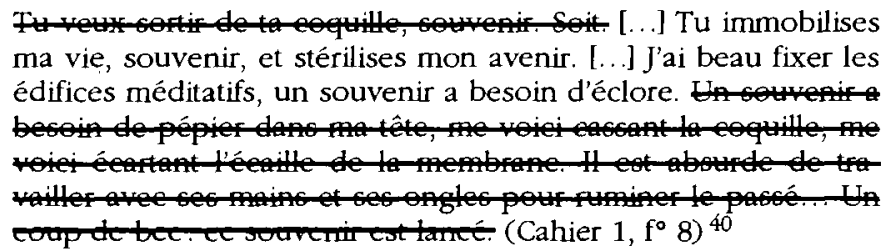

Ce souvenir qu'il s'agit d'extraire de la mémoire est significatif au point d'*immobiliser la vie" de la narratrice, de "stériliser son avenir" (Cahier 1, fo 8).

Ce souvenir se situe en fait bien avant la rencontre avec Isabelle, au début de la puberté, vers quatorze ans précise-t-elle. Le récit de ce fantasme érotique autour du sac d'un professeur Mademoiselle Godfroy - dans lequel la narratrice met la main, est décrit dans ce premier cahier de Ravages de manière très détaillée:

J'avais placé ma main dans l'objet indispensable de mademoiselle Godfroy. J'avais enfoncé ma main dans le trou de son argent, de sa poudre de riz. Ma main caressait le mouchoir, le porte-billet, le poudrier. Caresser, c'est faire l'inventaire superficiel de ce qu'on a ; caresser, c'est être possédé par ce qu'on croyait posséder dans la subtilité. [...] (Cahier 1, fo 15) ${ }^{41}$

Intégralement supprimé de Ravages, il sera cependant repris dans La bâtarde (p. 64-65), mais de manière brève et tout à fait anodine; l'aspect érotique en ayant été purement et simplement éliminé, il ne reste en fait que le "cadre" 42 .

40 Manuscrit inédit, reproduit avec l'autorisation de Carlo Jansiti. Les biffures sont de la main de Violette Leduc.

41 La page entière, ainsi que les autres pages relatant cet épisode, est biffée d'une croix de saint. André de la main de Simone de Beauvoir.

42 Il faudrait pouvoir également étudier et comparer la genèse de cet épisode dans les cahiers de La bâtarde (au nombre de cinquante-huit!), notamment 
Pourquoi cet épisode, qui semble tenir une place si importante dans les cahiers manuscrits de Ravages, a-t-il totalement disparu du texte imprimé? Il est vrai que les pages du début de ce cahier sont biffées d'une croix de saint André. Leur suppression est-elle de la main de Simone de Beauvoir, ou résulte-t-elle, plus probablement, d'une décision commune? L'enquête ne fait que commencer, il reste encore beaucoup à déchiffrer, à comparer, à interpréter... Seule est tangible, pour l'instant, la certitude qu'il existe un net décalage entre les manuscrits et le texte imprimé, et qu'un important travail de recherche reste à faire.

afin de voir si l'évolution génétique (suppression de l'aspect érotique) y est parallèle à celle dont témoignent les manuscrits de Ravages. Je n'ai pas encore eu accès à ces cahiers. 